\title{
PARACOMPACT SUBSPACES IN THE BOX PRODUCT TOPOLOGY
}

\author{
PETER NYIKOS AND LESZEK PIA̧TKIEWICZ
}

(Communicated by Franklin D. Tall)

\begin{abstract}
In 1975 E. K. van Douwen showed that if $\left(X_{n}\right)_{n \in \omega}$ is a family of Hausdorff spaces such that all finite subproducts $\prod_{n<m} X_{n}$ are paracompact, then for each element $x$ of the box product $\square_{n \in \omega} X_{n}$ the $\sigma$-product $\sigma(x)=$ $\left\{y \in \square_{n \in \omega} X_{n}:\{n \in \omega: x(n) \neq y(n)\}\right.$ is finite $\}$ is paracompact. He asked whether this result remains true if one considers uncountable families of spaces. In this paper we prove in particular the following result:
\end{abstract}

Theorem. Let $\kappa$ be an infinite cardinal number, and let $\left(X_{\alpha}\right)_{\alpha \in \kappa}$ be a family of compact Hausdorff spaces. Let $x \in \square=\square_{\alpha \in \kappa} X_{\alpha}$ be a fixed point. Given a family $\mathcal{R}$ of open subsets of $\square$ which covers $\sigma(x)$, there exists an open locally finite in $\square$ refinement $\mathcal{S}$ of $\mathcal{R}$ which covers $\sigma(x)$.

We also prove a slightly weaker version of this theorem for Hausdorff spaces with "all finite subproducts are paracompact" property. As a corollary we get an affirmative answer to van Douwen's question.

\section{INTRODUCTION}

A box product is a topological space which takes a Cartesian product of spaces for the point-set, and takes an arbitrary Cartesian product of open subsets for a base element. The box product topology is nontrivial in the case of infinitely many factor spaces, strictly stronger than the usual Tychonoff topology of pointwise convergence, because each factor of a basic open set is permitted to be a proper subset of a factor space.

In 1991 Brian Lawrence showed in [L] that (in ZFC) the box product $\square^{\omega_{1}}(\omega+1)$ of $\omega_{1}$ many copies of $\omega+1$ is neither normal nor collectionwise Hausdorff (and hence not paracompact), solving an old problem due to Arthur H. Stone $(1964,[K])$ and Mary Ellen Rudin $(1975,[R])$. In the same paper he proved that if $2^{\omega}=2^{\omega_{1}}$, then the $\Sigma$-product, that is,

$$
\left\{x \in \square^{\omega_{1}}(\omega+1):\left\{\alpha \in \omega_{1}: x(\alpha) \neq \omega\right\} \text { is countable }\right\},
$$

is nonnormal.

Received by the editors June 9, 1993.

1991 Mathematics Subject Classification. Primary 54D18; Secondary 54B10.

Key words and phrases. Paracompact space, box product.

The first author's research was supported in part by NSF Grant DMS-8901931. 
In this paper we consider $\sigma$-products, where for given $x$ in a box product $\square_{\alpha \in \varkappa} X_{\alpha}$ the $\sigma$-product is the set

$$
\sigma(x)=\left\{y \in \square_{\alpha \in \varkappa} X_{\alpha}:\{\alpha \in \varkappa: y(\alpha) \neq x(\alpha)\} \text { is finite }\right\} .
$$

We prove (in ZFC) that if $\left(X_{\alpha}\right)_{\alpha \in \varkappa}$ is a family of topological spaces, such that $\prod_{\alpha \in \theta} X_{\alpha}$ is paracompact Hausdorff for every finite $\theta \subseteq \varkappa$, then $\sigma(x)$ is paracompact for each $x \in \square_{\alpha \in \varkappa} X_{\alpha}$. This answers affirmatively a question asked by Eric van Douwen in $(1975,[\mathrm{vD}])$ (question 145 on van Mill's list of van Douwen's problems in $(1993,[\mathrm{vM}]))$.

If each $X_{\alpha}$ is a compact Hausdorff space, we get the stronger result stated in the abstract. This result is a generalization of a theorem of Scott W. Williams $(1990,[W])$, who has a similar result for compact metrizable spaces.

\section{Notation AND TERMinOlOGY}

Let $X$ be a topological space. $\mathcal{P}(X)$ denotes the power set of $X$. For each $A \subseteq X, \bar{A}$ denotes the closure of $A$ in $X$, and $\operatorname{int}(A)$ denotes the interior of $A$ in $X$. The family $\mathcal{W}$ of subsets of $X$ is a cover (or covering) of $X$ if $\cup \mathcal{W}=X . \mathcal{W}$ is an open covering of $X$ if each set in $\mathcal{W}$ is an open subset of $X . \mathcal{W}$ is an irreducible open covering of $X$ if $\mathcal{W}$ is an open covering of $X$ which is irreducible, that is, no proper subfamily of $\mathcal{W}$ covers $X$.

For given infinite cardinal $\varkappa$ and a family of topological spaces $\left\{X_{\alpha}: \alpha \in \varkappa\right\}$, $\square=\square_{\alpha \in \varkappa} X_{\alpha}$ denotes the box product of $X_{\alpha}$ 's. If $\lambda \subseteq \varkappa$, then Proj $\square_{\square \lambda}$ is the projection from $\square_{\alpha \in \varkappa} X_{\alpha}$ onto $\square_{\alpha \in \lambda} X_{\alpha}$. If $x \in \square$, then a box neighborhood of $x$ is any set of the form $\prod_{\alpha \in \varkappa} A_{\alpha}$ where $x(\alpha) \in \operatorname{int}\left(A_{\alpha}\right)$ for each $\alpha \in \varkappa$. The box neighborhood $\prod_{\alpha \in \varkappa} A_{\alpha}$ is open if each $A_{\alpha}$ is an open subset of $X_{\alpha}$.

The $\sigma$-product $\sigma(x)$ is the following set:

$$
\sigma(x)=\{y \in \square:\{\alpha \in \varkappa: x(\alpha) \neq y(\alpha)\} \text { is finite }\} .
$$

$[\varkappa]^{<\omega}$ is the family of all finite subsets of $\varkappa$. Let $\mathcal{T}$ and $\mathcal{S}$ be two families of subsets of $\square$. We say that $\mathcal{T}$ refines $\mathcal{S}$ if for each $T \in \mathcal{T}$ there is $S \in \mathcal{S}$ such that $T \subseteq S$; we say that $\mathcal{T}$ strictly refines $\mathcal{S}$ if for each $T \in \mathcal{T}$ there is $S \in \mathcal{S}$ such that $\bar{T} \subseteq S$. Note that we do not require that $\bigcup \mathcal{T}=\bigcup \mathcal{S}$. For every finite $\lambda \subseteq \varkappa$ we will use the following notation:

$$
\begin{aligned}
\square^{\lambda} & =\square_{\alpha \in \lambda} X_{\alpha}, \\
\text { for each } y \in \square, y^{\lambda} & =\operatorname{Proj}_{\square \lambda}(y), \\
\text { for } A \subseteq \square, A^{\lambda} & =\left\{y^{\lambda}: y \in A\right\}, \\
\text { for } \mathcal{A} \subseteq \mathcal{P}(\square), \mathcal{A}^{\lambda} & =\left\{A^{\lambda}: A \in \mathcal{A}\right\} .
\end{aligned}
$$

\section{The PARACOMPACT CASE}

Here is our main result.

Theorem 2.1. Let $\varkappa$ be an infinite cardinal and let $\left\{X_{\alpha}: \alpha \in \varkappa\right\}$ be a family of spaces such that $\square^{\lambda}$ is paracompact Hausdorff for each $\lambda \in[\varkappa]^{<\omega}$. Let $x \in \square=$ $\square_{\alpha \in \varkappa} X_{\alpha}$ be a fixed point and let $\mathcal{R}$ be a family of open sets in $\square$ which covers $\sigma(x)$. 
There exist an open in $\square$ neighborhood $U$ of $\sigma(x)$ and a locally finite in $U$ family $\mathcal{B}$ of open sets, such that $\mathcal{B}$ strictly refines $\mathcal{R}$ and $\bigcup \mathcal{B}=U$.

Note that the conclusion is strictly stronger than the claim that $\sigma(x)$ is paracompact. For example, a discrete space is paracompact, yet even a closed discrete subspace of a regular space need not have the property in the conclusion. Indeed, if one covers the $x$-axis in the tangent disk space with basic tangent disk neighborhoods at each point, no such $U$ or $\mathcal{B}$ exist.

Before proving the theorem we first prove a rather technical lemma. In it, we produce open covers of $\sigma(x)$ by induction: first of $x$ itself, then all points that deviate from it in exactly one coordinate, then two coordinates, and so forth. At the $n$th stage of the induction, we take each $n$-element subset $\lambda$ of $\varkappa$, and handle all the points which deviate from $x$ at exactly the coordinates indexed by $\lambda$. This set of points is homeomorphic to $\square^{\lambda}$ so a lot of our attention is focused on producing the projections $\left(\mathcal{W}_{\lambda}\right)^{\lambda},\left(\mathcal{V}_{\lambda}\right)^{\lambda}$ and $\left(\mathcal{U}_{\lambda}\right)^{\lambda}$ of the covers $\mathcal{W}_{\lambda}, \mathcal{V}_{\lambda}$ and $\mathcal{U}_{\lambda}$ for this set, which we identify with $\square^{\lambda} \times\left\{x^{\varkappa-\lambda}\right\}$.

Lemma 2.2. Under the assumptions of Theorem 2.1, there are collections

$$
\mathcal{W}=\bigcup\left\{\mathcal{W}_{\lambda}^{\#}: \lambda \in[\varkappa]^{<\omega}\right\}, \quad \mathcal{U}=\bigcup\left\{\mathcal{U}_{\lambda}: \lambda \in[\varkappa]^{<\omega}\right\}
$$

and

$$
\mathcal{V}=\bigcup\left\{\mathcal{V}_{\lambda}: \lambda \in[\varkappa]^{<\omega}\right\},
$$

of open subsets of $\square$ subject to the following conditions for all finite $\lambda \subseteq \varkappa$ :

(A) (a0) If $\mathcal{W}_{\lambda}=\bigcup\left\{\mathcal{W}_{\tau}^{\#}: \tau \subseteq \lambda\right\}$, then $\left(\mathcal{W}_{\lambda}\right)^{\lambda}$ is a locally finite open cover of $\square^{\lambda}$, such that for each $W \in \mathcal{W}_{\lambda}$ :

(a1) There exists $R \in \mathcal{R}$, such that $\bar{W} \subseteq R$,

(a2) $W=W^{\lambda} \times W^{\varkappa-\lambda}$ where $W^{\varkappa-\lambda}$ is an open box neighborhood of $x^{\varkappa-\lambda}$.

For each $W \in \mathcal{W}_{\lambda}$ we choose an open box neighborhood $U(W)^{\varkappa-\lambda}$ of $x^{\varkappa-\lambda}$, so that:

(a3) $\overline{U(W)}^{\varkappa-\lambda} \subseteq W^{\varkappa-\lambda}$.

(B) (b0) $\left(\mathcal{U}_{\lambda}\right)^{\lambda}$ is a locally finite open cover of $\square^{\lambda}$ and the following conditions are satisfied for each $U \in \mathcal{U}_{\lambda}$ :

(b1) There exists $W \in \mathcal{W}_{\lambda}$, such that $\bar{U} \subseteq W$,

(b2) $U=U^{\lambda} \times U^{\varkappa-\lambda}$,

(b3) $\mathcal{S T}_{\mathcal{W}}(U)=\left\{W \in \mathcal{W}_{\lambda}: U^{\lambda} \cap W^{\lambda} \neq \varnothing\right\}$ is a finite set,

(b4) $U^{\varkappa-\lambda}=\bigcap\left\{U(W)^{\varkappa-\lambda}: W \in \mathcal{S T}_{\mathcal{W}}(U)\right\}$.

(C) (c0) $\left(\mathcal{V}_{\lambda}\right)^{\lambda}$ is a locally finite open cover of $\square^{\lambda}$ and the following conditions are satisfied for each $V \in \mathcal{V}_{\lambda}$ :

(c1) There exists $U \in \mathcal{U}_{\lambda}$, such that $V \subseteq U$,

(c2) $V=V^{\lambda} \times V^{\varkappa-\lambda}$,

(c3) $\mathcal{S T}_{\mathcal{U}}(V)=\left\{U \in \mathcal{U}_{\lambda}: V^{\lambda} \cap U^{\lambda} \neq \varnothing\right\}$ is a finite set,

(c4) $V^{\varkappa-\lambda}=\bigcap\left\{U^{\varkappa-\lambda}: U \in \mathcal{S T}_{\mathcal{U}}(V)\right\}$.

Let's note that (b3) together with (c3) give us:

(c5) $\mathcal{S T}^{2}(V)=\bigcup\left\{\mathcal{S} \mathcal{T}_{\mathcal{W}}(U): U \in \mathcal{S T}_{\mathcal{U}}(V)\right\}$ is finite, for each $V \in \mathcal{V}_{\lambda}$.

(D) For each $W_{0} \in \mathcal{W}_{\lambda}^{\#}$ and each $\theta \subsetneq \lambda$, there exists $V_{0} \in \mathcal{V}_{\theta}$, such that the following conditions hold:

(d1) $\left(W_{0}\right)^{\theta} \subseteq\left(V_{0}\right)^{\theta}$, 
(d2) $\left(W_{0}\right)^{\lambda-\theta}$ is disjoint from $\bigcap\left\{\overline{U(W)}^{\lambda-\theta}: W \in \mathcal{S} \mathcal{T}_{\mathcal{W}}\left(V_{0}\right)\right\}$,

(d3) $\left(W_{0}\right)^{\varkappa-\lambda} \subseteq \bigcap\left\{U(W)^{\varkappa-\lambda}: W \in \mathcal{S T}^{2}\left(V_{0}\right)\right\}$.

Proof of Lemma 2.2. We construct families

$$
\mathcal{W}=\bigcup\left\{\mathcal{W}_{\lambda}^{\#}: \lambda \in[\varkappa]^{<\omega}\right\}, \quad \mathcal{U}=\bigcup\left\{\mathcal{U}_{\lambda}: \lambda \in[\varkappa]^{<\omega}\right\}
$$

and

$$
\mathcal{V}=\bigcup\left\{\mathcal{V}_{\lambda}: \lambda \in[\varkappa]^{<\omega}\right\}
$$

by induction on $n=|\lambda|$. For $n=0$ choose any open box neighborhoods $W_{0}$ and $U\left(W_{0}\right)$ of $x$, so that $\overline{U\left(W_{0}\right)} \subseteq W_{0}$ and $\overline{W_{0}} \subseteq R$, for some $R \in \mathcal{R}$. We put $\mathcal{W}_{\varnothing}^{\#}=\left\{W_{0}\right\}$ and $\mathcal{U}_{\varnothing}=\mathcal{V}_{\varnothing}=\left\{U\left(W_{0}\right)\right\}$. Clearly all conditions in (A)-(D) are satisfied.

Now assume that for some natural number $n,\left\{\mathcal{W}_{\theta}^{\#}, \mathcal{U}_{\theta}, \mathcal{V}_{\theta}\right\}_{|\theta| \leq n}$ have been constructed so that all the conditions in (A)-(D) hold, and let $\lambda \in[\varkappa]^{n+1}$. For each $\theta \subsetneq \lambda$ let

$$
\mathcal{F}_{\theta}=\left\{\bar{V}^{\theta} \times \bigcap\left\{\overline{U(W)}^{\lambda-\theta}: W \in \mathcal{S} \mathcal{T}_{\mathcal{W}}(V)\right\}: V \in \mathcal{V}_{\theta}\right\}
$$

and let

$$
F=\bigcup\left\{\bigcup \mathcal{F}_{\theta}: \theta \subsetneq \lambda\right\}
$$

By (c0) for $\theta \subsetneq \lambda$ we get: each $\mathcal{F}_{\theta}$ is a locally finite family of closed subsets of $\square^{\lambda}$.

By (c1),(b1),(a3) for $\theta \subsetneq \lambda$ and the definition of $\mathcal{S T}_{\mathcal{W}}$ in (b3) we get:

$$
\text { For each } \theta \subsetneq \lambda, \bigcup \mathcal{F}_{\theta} \subseteq \bigcup\left(\mathcal{W}_{\theta}\right)^{\lambda} \text {. }
$$

Now (2),(3) and (4) together with the definition of $\mathcal{W}_{\theta}$ in (a0) give us:

$$
F \text { is a closed subset of } \square^{\lambda} \text { contained in } \bigcup\left\{\bigcup\left(\mathcal{W}_{\theta}^{\#}\right)^{\lambda}: \theta \subsetneq \lambda\right\} .
$$

For each $\theta \subsetneq \lambda$ let

$$
\mathcal{O}_{\theta}=\left\{V^{\theta} \times \square^{\lambda-\theta}: V \in \mathcal{V}_{\theta}\right\}
$$

By (c0) for $\theta \subsetneq \lambda$ each $\mathcal{O}_{\theta}$ is an open covering of $\square^{\lambda}$. Let

$$
X=\square^{\lambda} \backslash\left\{\bigcup\left(\mathcal{W}_{\theta}^{\#}\right)^{\lambda}: \theta \subsetneq \lambda\right\} .
$$

$F$ and $X$ are disjoint closed subsets (see (5) and (7)) of a paracompact space $\square^{\lambda}$, hence there exists a family $\left(\mathcal{W}_{\lambda}^{\#}\right)^{\lambda}$ of open subsets of $\square^{\lambda}$, such that:

$$
\text { For each } \theta \subsetneq \lambda,\left(\mathcal{W}_{\lambda}^{\#}\right)^{\lambda} \text { is a locally finite refinement of } \mathcal{O}_{\theta} \text {, }
$$




$$
\left(\mathcal{W}_{\lambda}^{\#}\right)^{\lambda} \text { covers } X
$$

$$
\forall W^{\lambda} \in\left(\mathcal{W}_{\lambda}^{\#}\right)^{\lambda} \exists R_{W} \in \mathcal{R} \text { such that } x^{\varkappa-\lambda} \in R_{W}^{\varkappa-\lambda} \text { and } \overline{W^{\lambda}} \subseteq R_{W}^{\lambda} .
$$

For each $W^{\lambda} \in\left(\mathcal{W}_{\lambda}^{\#}\right)^{\lambda}$ we choose an open box neighborhood $W^{\varkappa-\lambda}$ of $x^{\varkappa-\lambda}$, so that:

$$
\overline{W^{\varkappa-\lambda}} \subseteq R_{W}^{\varkappa-\lambda} \cap \bigcap\left\{V^{\varkappa-\lambda}: V \in \mathcal{V}_{\theta}, \theta \subsetneq \lambda \text { and }\left(W^{\lambda}\right)^{\theta} \subseteq V^{\theta}\right\}
$$

and choose an open box neighborhood $U(W)^{\varkappa-\lambda}$ of $x^{\varkappa-\lambda}$ so that

$$
\overline{U(W)}^{\varkappa-\lambda} \subseteq W^{\varkappa-\lambda}
$$

Let

$$
\mathcal{W}_{\lambda}^{\#}=\left\{W^{\lambda} \times W^{\varkappa-\lambda}: W^{\lambda} \in\left(\mathcal{W}_{\lambda}^{\#}\right)^{\lambda}\right\}
$$

Note that the family $\left(\mathcal{W}_{\lambda}^{\#}\right)^{\lambda}$ was already chosen (see $\left.8(\mathrm{a}-\mathrm{d})\right)$, and that $W^{\varkappa-\lambda}$ has been chosen for each $W^{\lambda} \in\left(\mathcal{W}_{\lambda}^{\#}\right)^{\lambda}$ (see (9)), so this is not circular.

Let us check that $\left(\mathcal{W}_{\lambda}\right)^{\lambda}=\bigcup\left\{\left(\mathcal{W}_{\theta}^{\#}\right)^{\lambda}: \theta \subseteq \lambda\right\}$ satisfies the conditions in (A) and (D).

First (8a) together with $(\mathrm{a} 0)$ for $\theta \subsetneq \lambda$ give us that $\left(\mathcal{W}_{\lambda}\right)^{\lambda}$ is locally finite, while (7) and (8b) imply that it covers $\square^{\lambda}$, hence (a0) holds. Now (8d),(9) and (11) imply (a1), (11) implies (a2) and (10) gives us (a3).

Next (6) and (8a) imply (d1). By (1), (2) and (8c) we get (d2), while (9) and (d1) together with conditions (b4) and (c4) for $\theta \subsetneq \lambda$ and the definition of $\mathcal{S T}^{2}$ in (c5) give us (d3). Thus all the conditions in (A) and (D) are satisfied.

Finally since $\left(\mathcal{W}_{\lambda}\right)^{\lambda}$ is a locally finite open covering of a paracompact space $\square^{\lambda}$, we can choose open locally finite families $\mathcal{U}_{\lambda}$ and $\mathcal{V}_{\lambda}$, so that all conditions in (B) and $(\mathrm{C})$ are also satisfied.

We are ready now to prove the theorem.

Proof of Theorem 2.1. Let $\mathcal{T}$ be a family of open sets in $\square$ which covers $\sigma(x)$ and strictly refines $\mathcal{R}$.

We construct families $\mathcal{W}, \mathcal{U}$ and $\mathcal{V}$ for $\mathcal{T}$ as in Lemma 2.2.

Lemma A. $\forall \lambda, \theta \in[\varkappa]^{<\omega}$ if $\lambda \nsubseteq \theta$ and $\theta \nsubseteq \lambda$, then $\bigcup \mathcal{W}_{\lambda}^{\#} \cap \bigcup \mathcal{W}_{\theta}^{\#}=\varnothing$.

Proof of Lemma A. Assume that $\lambda, \theta \in[\varkappa]^{<\omega}$ are such that $\lambda \nsubseteq \theta$ and $\theta \nsubseteq \lambda$.

Let $W_{1} \in \mathcal{W}_{\lambda}^{\#}, W_{2} \in \mathcal{W}_{\theta}^{\#}$ and suppose that:

$$
W_{1} \cap W_{2} \neq \varnothing .
$$

Let $\delta=\lambda \cap \theta$. Since $\delta \subsetneq \lambda$ and $\delta \subsetneq \theta$, we can choose $V_{1}, V_{2} \in \mathcal{V}_{\delta}$, so that $W_{1}^{\delta} \subseteq V_{1}^{\delta}, W_{2}^{\delta} \subseteq V_{2}^{\delta}$ and the conditions (d2) and (d3) are satisfied (see (D) in 
Lemma 2.2). Clearly $V_{1}^{\delta} \cap V_{2}^{\delta} \neq \varnothing$. Since $\mathcal{V}$ refines $\mathcal{U}$ we get (see the definitions of $\mathcal{S} \mathcal{T}_{\mathcal{W}}$ and $\mathcal{S T}^{2}$ in (c3) and (c5) in Lemma 2.2):

$$
\mathcal{S T}_{\mathcal{W}}\left(V_{1}\right) \subseteq \mathcal{S T}^{2}\left(V_{2}\right)
$$

By (d2) in Lemma 2.2 we get:

$$
\left(W_{1}\right)^{\lambda-\delta} \text { is disjoint from } \bigcap\left\{\overline{U(W)}^{\lambda-\delta}: W \in \mathcal{S T}_{\mathcal{W}}\left(V_{1}\right)\right\} .
$$

Since $(\lambda-\delta) \subseteq(\varkappa-\theta),($ A2) above and (d3) in Lemma 2.2 imply that:

$$
\begin{aligned}
\left(W_{2}\right)^{\lambda-\delta} & \subseteq \bigcap\left\{U(W)^{\lambda-\delta}: W \in \mathcal{S T}^{2}\left(V_{2}\right)\right\} \\
& \subseteq \bigcap\left\{U(W)^{\lambda-\delta}: W \in \mathcal{S T}_{\mathcal{W}}\left(V_{1}\right)\right\} .
\end{aligned}
$$

By (A3) and (A4) we get $\left(W_{1}\right)^{\lambda-\delta} \cap\left(W_{2}\right)^{\lambda-\delta}=\varnothing$ which contradicts (A1).

The following part of the proof mimics a well-known argument, used to show that if every open cover of a regular space has a $\sigma$-locally finite open refinement which covers the space, then every open cover of the space has a locally finite closed refinement which covers the space. See for example [E].

For each $n \in \omega$ put:

$$
\begin{gathered}
\mathcal{W}_{n}=\bigcup\left\{\mathcal{W}_{\lambda}:|\lambda|=n\right\}, \\
O_{n}=\bigcup \mathcal{W}_{n} \backslash \bigcup\left\{\bigcup \mathcal{W}_{m}: m<n\right\},
\end{gathered}
$$

and

$$
\mathcal{F}_{n}=\left\{\overline{W \cap O_{n}}: W \in \mathcal{W}_{n}\right\}
$$

Put

$$
\mathcal{F}=\bigcup\left\{\mathcal{F}_{n}: n \in \omega\right\} .
$$

Note that (see (a1) in Lemma 2.2)

$$
\forall F=\overline{W \cap O_{n}} \in \mathcal{F} \exists T_{F} \in \mathcal{T} \text { such that } F \subseteq \bar{W} \subseteq T_{F} .
$$

Clearly (see (b0),(b1) in Lemma 2.2 and (1),(2) above)

$$
\sigma(x) \subseteq \bigcup \mathcal{U} \subseteq \bigcup \mathcal{W} \subseteq \bigcup \mathcal{F}
$$

Lemma B. Each $U \in \mathcal{U}=\bigcup\left\{\mathcal{U}_{\theta}: \theta \in[\varkappa]^{<\omega}\right\}$ meets only finitely many sets in $\mathcal{F}$.

Proof of Lemma B. Fix $U_{0} \in \mathcal{U}_{\theta}$ for some $\theta \in[\varkappa]^{<\omega}$. Since $\mathcal{U}_{\theta}$ refines $\mathcal{W}_{\theta}=$ $\bigcup\left\{\mathcal{W}_{\tau}^{\#}: \tau \subseteq \theta\right\}$, we can choose $W_{0} \in \mathcal{W}_{\tau_{0}}^{\#}$ for some $\tau_{0} \subseteq \theta$, so that:

$$
U_{0} \subseteq W_{0} .
$$


Let $m=\left|\tau_{0}\right|$, and let $F \in \mathcal{F}$ meet $U_{0}$,

$$
F \cap U_{0} \neq \varnothing \text {. }
$$

Say $F=\overline{W \cap O_{n}}$ for some $W \in \mathcal{W}_{\lambda_{0}}^{\#}$ with $\left|\lambda_{0}\right|=n$. Since $U_{0}$ is an open subset of $\square$, (B2) above implies that $W \cap O_{n} \cap U_{0} \neq \varnothing$, hence $O_{n} \cap U_{0} \neq \varnothing$ and by (B1) we get:

$$
O_{n} \cap W_{0} \neq \varnothing .
$$

Also $W \cap U_{0} \neq \varnothing$ and again by (B1) we have:

$$
W \cap W_{0} \neq \varnothing .
$$

Now (1) and (B3) imply that $n \leq m$, while (B4) implies that $\tau_{0} \subseteq \lambda_{0}$ or $\lambda_{0} \subseteq \tau_{0}$ (see Lemma A). Thus $\lambda_{0} \subseteq \tau_{0} \subseteq \theta$ and the following inclusions hold:

$$
\begin{aligned}
& \left\{F=\overline{W \cap O_{n}}: F \cap U_{0} \neq \varnothing\right\} \\
\subseteq & \left\{F=\overline{W \cap O_{n}}: n \leq m, W \in \mathcal{W}_{\lambda}^{\#} \text { with }|\lambda|=n, \lambda \subseteq \theta, \text { and } U_{0} \cap W \neq \varnothing\right\} \\
\subseteq & \left\{F=\overline{W \cap O_{n}}: n \leq m, W \in \mathcal{W}_{\theta} \text { and } U_{0}^{\theta} \cap W^{\theta} \neq \varnothing\right\} .
\end{aligned}
$$

By (b3) in Lemma 2.2 the last set is finite, hence the first set is finite as well.

The following part of the proof mimics a standard argument, used to show that if every open cover of a regular space has a locally finite closed refinement which covers the space, then the space is paracompact (see [E]).

$\mathcal{U}$ is a family of open subsets of $\square$ with $\sigma(x) \subseteq \bigcup \mathcal{U}$, so we can apply Lemma 2.2 to $\mathcal{U}$ to get families $\mathcal{W}^{\prime}, \mathcal{U}^{\prime}, \mathcal{V}^{\prime}$ and then $\mathcal{F}^{\prime}$, just like $\mathcal{W}, \mathcal{U}, \mathcal{V}$ and $\mathcal{F}$ were constructed for $\mathcal{T}$. For each $F \in \mathcal{F}$ put

$$
F^{*}=\bigcup \mathcal{U}^{\prime} \backslash \bigcup\left\{F^{\prime} \in \mathcal{F}^{\prime}: F^{\prime} \cap F=\varnothing\right\},
$$

and let

$$
\mathcal{F}^{*}=\left\{F^{*}: F \in \mathcal{F}\right\} .
$$

Note that for each $F \in \mathcal{F}$ the following inclusions hold:

$$
\bigcup \mathcal{U}^{\prime} \cap F \subseteq F^{*} \subseteq \bigcup \mathcal{U}^{\prime}
$$

and since $\mathcal{U}^{\prime}$ refines $\mathcal{U}$, we get that (see (4)) $\bigcup \mathcal{U}^{\prime} \subseteq \cup \mathcal{F}$, hence

$$
\bigcup \mathcal{F}^{*}=\bigcup \mathcal{U}^{\prime}
$$

By Lemma B we get $\left\{F^{\prime} \cap \bigcup \mathcal{U}^{\prime}: F^{\prime} \in \mathcal{F}^{\prime}\right\}$ is a locally finite in $\bigcup \mathcal{U}^{\prime}$ family of closed subsets of $\bigcup \mathcal{U}^{\prime}$, hence (see (5))

$$
\mathcal{F}^{*} \text { is an open cover of } \bigcup \mathcal{U}^{\prime} \text {. }
$$


Lemma C. $\mathcal{F}^{*}$ is locally finite in $\bigcup \mathcal{U}^{\prime}$.

Proof of Lemma C. Let $U^{\prime} \in \mathcal{U}^{\prime}$. We will show that $U^{\prime}$ meets only finitely many sets in $\mathcal{F}^{*}$. First let us notice that Lemma B implies that $U^{\prime}$ meets only finitely many elements of $\mathcal{F}^{\prime}$. Let

$$
\left\{F_{1}^{\prime}, F_{2}^{\prime}, \ldots, F_{n}^{\prime}\right\}=\left\{F^{\prime} \in \mathcal{F}^{\prime}: F^{\prime} \cap U^{\prime} \neq \varnothing\right\} .
$$

Note that $\bigcup \mathcal{U}^{\prime} \subseteq \bigcup \mathcal{F}^{\prime}$ (see (4)), hence

$$
U^{\prime} \subseteq \bigcup\left\{F_{i}^{\prime}: i \leq n\right\} .
$$

For each $i \leq n$ fix $U_{i} \in \mathcal{U}$, so that (see (3))

$$
F_{i}^{\prime} \subseteq U_{i}
$$

For each $F^{*} \in \mathcal{F}^{*}$ we have:

$$
\begin{gathered}
U^{\prime} \cap F^{*} \neq \varnothing \\
\Longrightarrow \exists i \leq n F_{i}^{\prime} \cap F^{*} \neq \varnothing(\text { see }(\mathrm{C} 1)), \\
\Longrightarrow \exists i \leq n F_{i}^{\prime} \cap F \neq \varnothing(\text { see }(5)), \\
\Longrightarrow \exists i \leq n U_{i} \cap F \neq \varnothing(\text { see }(\mathrm{C} 2)) .
\end{gathered}
$$

Now Lemma B implies that each $\left\{F \in \mathcal{F}: U_{i} \cap F \neq \varnothing\right\}$ is finite, hence $\left\{F^{*} \in \mathcal{F}^{*}: U^{\prime} \cap F^{*} \neq \varnothing\right\}$ is a finite set as well.

For each $F \in \mathcal{F}$ choose $T_{F} \in \mathcal{T}$, so that $F \subseteq T_{F}$ (see (3)) and put $B_{F}=F^{*} \cap T_{F}$. Let $\mathcal{B}=\left\{B_{F}: F \in \mathcal{F}\right\}$. We will show that $\bigcup \mathcal{U}^{\prime}$ and $\mathcal{B}$ satisfy all the required conditions.

First since $\mathcal{U}^{\prime}$ was constructed for $\mathcal{U}$ in the same way as $\mathcal{U}$ was constructed for $\mathcal{T}$, (4) implies that $\sigma(x) \subseteq \cup \mathcal{U}^{\prime}$. It is also clear that $\mathcal{B}$ consists of open sets (see $(8))$.

Now since $\mathcal{T}$ strictly refines $\mathcal{R}$ we get

$$
\forall B_{F}=F^{*} \cap T_{F} \in \mathcal{B} \exists R \in \mathcal{R} \text { such that } \overline{B_{F}} \subseteq \overline{T_{F}} \subseteq R .
$$

Each $B_{F}$ is a subset of corresponding $F^{*}$ so Lemma $\mathrm{C}$ implies that the family $\mathcal{B}$ is locally finite in $\bigcup \mathcal{U}^{\prime}$.

Finally $\bigcup \mathcal{B} \subseteq \bigcup \mathcal{F}^{*}$ and since $\bigcup \mathcal{U}^{\prime} \cap F \subseteq F^{*} \cap T_{F}=B_{F}$ for each $F \in \mathcal{F}$ (see (3) and (6)), we get $\bigcup \mathcal{U}^{\prime} \cap \bigcup \mathcal{F} \subseteq \cup \mathcal{B} \subseteq \cup \mathcal{F}^{*}$. On the other hand, (7) and (4) imply that $\bigcup \mathcal{F}^{*}=\bigcup \mathcal{U}^{\prime} \subseteq \bigcup \mathcal{U} \subseteq \bigcup \mathcal{F}$ and so $\bigcup \mathcal{B}=\bigcup \mathcal{F}^{*}=\bigcup \mathcal{U}^{\prime}$.

This concludes the proof of Theorem 2.1.

As a corollary to Theorem 2.1 we obtain the following affirmative answer to van Douwen's question.

Corollary 2.3. If $\left(X_{\alpha}\right)_{\alpha \in \varkappa}$ is an uncountable family of spaces such that all finite subproducts are paracompact Hausdorff, then for each $x \in \square_{\alpha \in \varkappa} X_{\alpha}$ the $\sigma$-product, $\sigma(x)$, is paracompact. 


\section{The COMPACT CASE}

If each $X_{\alpha}$ is a compact Hausdorff space, we have a strengthening of Theorem 2.1 mentioned in the abstract. We also have the following nicer version of Lemma 2.2 .

Lemma 3.1. Under the assumptions of Theorem 2.1 if each $X_{\alpha}$ is a compact Hausdorff space, there are families

$$
\mathcal{U}=\left\{U_{\lambda}: \lambda \in[\varkappa]^{<\omega}\right\} \quad \text { and } \mathcal{V}=\left\{V_{\lambda}: \lambda \in[\varkappa]^{<\omega}\right\}
$$

of open box neighborhoods of $x$, and a collection

$$
\mathcal{W}=\left\{\mathcal{W}_{\lambda}^{\#}: \lambda \in[\varkappa]^{<\omega}\right\}
$$

of finite families of open boxes in $\square$, subject to the following conditions for all $\lambda \in[\varkappa]^{<\omega}:$

(i) $\mathcal{W}_{\lambda}=\bigcup\left\{\mathcal{W}_{\tau}^{\#}: \tau \subseteq \lambda\right\}$ is an irreducible open covering of $\square^{\lambda} \times x^{\varkappa-\lambda}$.

(ii) $\forall W \in \mathcal{W}_{\lambda}^{\#} \exists R \in \mathcal{R}$ such that $\bar{W} \subseteq R$.

(iii) $\forall \alpha \notin \lambda \quad \overline{U_{\lambda \cup\{\alpha\}}} \subseteq V_{\lambda} \subseteq \overline{V_{\lambda}} \subseteq U_{\lambda}$.

(iv) $\forall W \in W_{\lambda}^{\#}$

(a) $\forall \alpha \notin \lambda \quad W(\alpha)=U_{\lambda}(\alpha)$,

(b) $\forall \tau \subsetneq \lambda \quad \exists \beta \in \lambda-\tau$ such that $W(\beta) \cap \overline{V_{\tau}(\beta)}=\varnothing$.

Proof of Lemma 3.1. We construct families $\mathcal{U}, \mathcal{V}$, and $\mathcal{W}$ by induction on $n=|\lambda|$. For $n=0$ pick any open box neighborhoods of $x, U_{\varnothing}$ and $V_{\varnothing}$, so that $x \in V_{\varnothing} \subseteq$ $\overline{V_{\varnothing}} \subseteq U_{\varnothing} \subseteq \overline{U_{\varnothing}} \subseteq R$ for some $R \in \mathcal{R}$, and put $\mathcal{W}_{\varnothing}^{\#}=\left\{U_{\varnothing}\right\}$. Clearly all four conditions are satisfied.

Now assume that for some natural number $n,\left\{\mathcal{U}_{\tau}, \mathcal{V}_{\tau}, \mathcal{W}_{\tau}^{\#}\right\}_{|\tau| \leq n}$ have been constructed so that all four conditions (i) - (iv) hold, and let $\lambda \in[\varkappa]^{n+1}$. For each $\tau \subsetneq \lambda$ put

$$
F_{\tau}=\square^{\tau} \times \overline{V_{\tau}^{\lambda-\tau}} \times x^{\varkappa-\lambda} .
$$

Let

$$
F=\bigcup\left\{F_{\tau}: \tau \subsetneq \lambda\right\} .
$$

Clearly each $F_{\tau}$ is a compact subset of $\square$. For each $\tau \subsetneq \lambda$ we have $|\tau| \leq n$, hence the inductive assumption implies (see (i), (iva) and (iii)) that $F_{\tau} \subseteq \bigcup \mathcal{W}_{\tau}$. Thus

$$
F \text { is a compact subset of } \square \text { and } F \subseteq \bigcup\left\{\bigcup \mathcal{W}_{\tau}^{\#}: \tau \subsetneq \lambda\right\} \text {. }
$$

Choose a family $\mathcal{W}_{\lambda}^{\#}$ of open boxes in $\square$, so that:

$$
\forall W \in \mathcal{W}_{\lambda}^{\#} \exists R \in \mathcal{R} \text { such that } \bar{W} \subseteq R,
$$

$$
\bigcup \mathcal{W}_{\lambda}^{\#} \text { is disjoint from } F \text {, }
$$


and (see (i) and (1) above)

(4) $\mathcal{W}_{\lambda}=\mathcal{W}_{\lambda}^{\#} \cup \bigcup\left\{\mathcal{W}_{\tau}^{\#}: \tau \subsetneq \lambda\right\}$ is an irreducible open covering of $\square^{\lambda} \times x^{\varkappa-\lambda}$.

Clearly $\mathcal{W}_{\lambda}^{\#}$ is finite, hence we can assume without loss of generality that for some open box neighborhood $U_{\lambda}$ of $x$ with

$$
\overline{U_{\lambda}} \subseteq \bigcap\left\{V_{\tau}: \tau \subsetneq \lambda\right\},
$$

we have

$$
\text { for all } W \in \mathcal{W}_{\lambda}^{\#}, W^{\varkappa-\lambda}=U_{\lambda}^{\varkappa-\lambda} \text {. }
$$

Finally we choose any box neighborhood $V_{\lambda}$ of $x$, so that

$$
\overline{V_{\lambda}} \subseteq U_{\lambda}
$$

Now (4) implies (i), (2) implies (ii), (5) and (7) give us (iii), while (6) implies (iva) and (3) implies (ivb).

Now Lemma 3.1 enables us to prove the following result which is probably interesting in its own.

Lemma 3.2. Let $\varkappa$ be an infinite cardinal and let $X_{\alpha}$ be a compact Hausdorff space for each $\alpha \in \varkappa$. Let $x \in \square=\square_{\alpha \in \varkappa} X_{\alpha}$ and let $O$ be an open neighborhood of $\sigma(x)$. There exists an open neighborhood $P$ of $\sigma(x)$ such that $\bar{P} \subseteq O$.

Proof of Lemma 3.2. $\mathcal{R}=\{O\}$ is a 1-element open cover of $\sigma(x)$. Let $\mathcal{U}, \mathcal{V}$ and $\mathcal{W}$ be families of open boxes constructed for $\mathcal{R}$ as in the proof of Lemma 3.1. Put $P=\bigcup \bigcup \mathcal{W}$. Clearly $P$ is an open neighborhood of $\sigma(x)$ in $\square$.

Claim 3.2.1. $\bar{P} \subseteq O$.

Proof of the claim. Let $y \in \square \backslash O$. We will find an open neighborhood of $y$ disjoint from P. We put $\tau_{-1}=\varnothing$ and construct by induction on $n$ subsets $\left\langle\theta_{n}\right\rangle_{n<\Delta}$ and $\left\langle\tau_{n}\right\rangle_{n<\Delta}$ of $\varkappa$ and open box neighborhoods $\left\langle T_{n}\right\rangle_{n<\Delta}$ of $y$, for some $\Delta \in \omega+1$, until the first (if any) infinite $\theta_{n}$ is found.

For $n=0$ we put:

$$
\begin{aligned}
& \theta_{0}=\left\{\gamma \in \varkappa: y(\gamma) \notin \overline{U_{\varnothing}(\gamma)}\right\}, \\
& \tau_{0}=\theta_{0},
\end{aligned}
$$

and inductively if $\theta_{n}$ and $\tau_{n}$ are defined for some $n \in \omega$, and if $\theta_{n}$ is finite, we put:

$$
\begin{aligned}
& \theta_{n+1}=\left\{\gamma \notin \tau_{n}: y(\gamma) \notin \overline{U_{\tau_{n}}(\gamma)}\right\}, \\
& \tau_{n+1}=\tau_{n} \cup \theta_{n+1} .
\end{aligned}
$$

Notice that

$$
\forall n<\Delta \tau_{n}=\bigcup_{m \leq n} \theta_{m} \text { and } \theta_{n} \cap \tau_{n-1}=\varnothing
$$


Note also that

$$
\forall n<\Delta \theta_{n} \neq \varnothing
$$

To see (4) choose $W \in \mathcal{W}_{\tau_{n-1}}^{\#}$ so that $y^{\tau_{n-1}} \in W^{\tau_{n-1}}$. Since $y \notin \bar{W}$ and (compare (iva) in Lemma 3.1) $\forall \alpha \notin \tau_{n-1} W(\alpha)=U_{\tau_{n-1}}(\alpha)$, there exists $\gamma \notin \tau_{n-1}$ such that $y(\gamma) \notin \overline{U_{\tau_{n-1}}(\gamma)}$. Clearly $\gamma \in \theta_{n}$.

Since we stop our inductive construction at the finite stage $n$ only when we get an infinite $\theta_{n},(4)$ implies that

$$
\Theta=\bigcup_{n<\Delta} \theta_{n} \text { is infinite . }
$$

Now, for each $n<\Delta$, that is, for each $n$ for which $\theta_{n}$ and $\tau_{n}$ are defined, we define $T_{n}$ as follows:

$$
\forall \alpha \in \varkappa
$$

$$
\begin{aligned}
& T_{n}(\alpha)=X_{\alpha} \backslash \overline{U_{\tau_{m-1}}} \text { if } \alpha \in \theta_{m} \text { for some } m \leq n, \\
& T_{n}(\alpha)=X_{\alpha} \text { if } \alpha \in \varkappa \backslash \bigcup_{m \leq n} \theta_{m} .
\end{aligned}
$$

Subclaim. $T=\bigcap\left\{T_{n}: n<\Delta\right\}$ is an open box neighborhood of $y$ disjoint from $P$. Proof of the subclaim. By (6) we get:

$$
\begin{gathered}
\forall n \forall \gamma \in \theta_{n} T(\gamma)=T_{n}(\gamma), \\
\forall \gamma \notin \Theta T(\gamma)=X_{\gamma},
\end{gathered}
$$

and since $\forall n<\Delta y \in T_{n}, T$ is an open box neighborhood of $y$. Next

$$
\forall \lambda \in[\varkappa]^{<\omega} \forall n \in \omega \forall W \in \mathcal{W}_{\lambda}^{\#} \in \mathcal{W} \text { if } W \cap T_{n} \neq \varnothing, \text { then } \tau_{n} \subseteq \lambda .
$$

We prove (8) by induction on $n$. For $n=0$ and each $\alpha \in \varkappa$ we have (see (iii),(iva) in Lemma 3.1 and (1))

$$
\begin{aligned}
& \alpha \notin \lambda \longrightarrow W(\alpha)=U_{\lambda}(\alpha) \subseteq U_{\varnothing}(\alpha), \\
& \alpha \in \theta_{0} \longrightarrow W(\alpha) \backslash \overline{U_{\varnothing}(\alpha)} \neq \varnothing .
\end{aligned}
$$

Therefore $\alpha \in \theta_{0} \longrightarrow \alpha \in \lambda$ and $\tau_{0}=\theta_{0} \subseteq \lambda$.

Now assume that (8) holds for some $n \in \omega$ and let $W \in W_{\lambda}^{\#}$ meet $T_{n+1}$. $W \cap$ $T_{n+1} \neq \varnothing \longrightarrow W \cap T_{n} \neq \varnothing$ and by the induction hypothesis we get $\tau_{n} \subseteq \lambda$. For each $\alpha \in \varkappa$ we have (see (iii),(iva) in Lemma 3.1 and (2))

$$
\begin{aligned}
\alpha \notin \lambda & \longrightarrow W(\alpha)=U_{\lambda}(\alpha) \subseteq U_{\tau_{n}}(\alpha), \\
\alpha \in \theta_{n+1} & \longrightarrow W(\alpha) \backslash \overline{U_{\tau_{n}}(\alpha)} \neq \varnothing .
\end{aligned}
$$

Therefore $\alpha \in \theta_{n+1} \longrightarrow \alpha \in \lambda$ and $\tau_{n+1}=\tau_{n} \cup \theta_{n+1} \subseteq \lambda$. This concludes the proof of (8).

By (8) if $W \in \mathcal{W}_{\lambda}^{\#} \in \mathcal{W}$ meets $T=\bigcap\left\{T_{n}: n<\Delta\right\}$, then $\Theta \subseteq \lambda$. It is clearly impossible since $\lambda$ is finite and $\Theta$ is infinite (see (5)). Hence $T \cap \bigcup \bigcup \mathcal{W}=\varnothing$ and the proof of the subclaim is completed.

By the subclaim $y \notin \bar{P}$, and since $y$ was an arbitrary point in $\square \backslash O$ we get $\bar{P} \subseteq O$. This completes the proof of the claim and the proof of the lemma.

Using the last lemma and Theorem 2.1 we easily get the theorem mentioned in the abstract. 
Theorem 3.3. Let $\varkappa$ be an infinite cardinal and let $\left\{X_{\alpha}: \alpha \in \varkappa\right\}$ be a family of compact Hausdorff spaces. Let $x \in \square=\square_{\alpha \in \varkappa} X_{\alpha}$ be a fixed point and let $\mathcal{R}$ be a family of open sets in $\square$ which covers $\sigma(x)$. There exists an open locally finite in $\square$ family $\mathcal{S}$ which strictly refines $\mathcal{R}$ and covers $\sigma(x)$.

Proof of Theorem 3.3. Let $U$ be an open neighborhood of $\sigma(x)$ and let $\mathcal{B}$ be a locally finite in $U$ family of open sets which strictly refines $\mathcal{R}$ and covers $U$ (see Theorem 2.1). By Lemma 3.2 we can choose an open neighborhood $V$ of $\sigma(x)$ so that $\bar{V} \subseteq U$. Put $\mathcal{S}=\{B \cap V: B \in \mathcal{B}\}$. Clearly $\mathcal{S}$ is a family of open subsets of $\square$ which strictly refines $\mathcal{R}$ and covers $\sigma(x)$. $\mathcal{S}$ is locally finite in $U$ and $\bigcup \mathcal{S}$ is disjoint from $\square \backslash \bar{V}$, hence $\mathcal{S}$ is locally finite in $U \cup(\square \backslash \bar{V})=\square$.

Question 3.4. Does Theorem 3.3 (equivalently Lemma 3.2) remain true if one drops the assumption of compactness of $X_{\alpha}$ 's and assumes only that each finite product of $X_{\alpha}$ 's is paracompact Hausdorff? In other words, is the natural common generalization of Theorems 2.1 and 3.3 valid?

Recall that the $\Sigma$-product is the subspace of $\square^{\omega_{1}}(\omega+1)$ defined as follows:

$$
\left\{x \in \square^{\omega_{1}}(\omega+1):\left\{\alpha \in \omega_{1}: x(\alpha) \neq \omega\right\} \text { is countable }\right\} .
$$

As stated in the introduction the $\Sigma$-product is consistently nonnormal. The following question remains open:

Question 3.5. Is the $\Sigma$-product nonnormal in ZFC?

\section{REFERENCES}

[vD] E. K. van Douwen, The box product of countably many metrizable spaces need not be normal, Fund. Math. 88 (1975), 127-132. MR 52:6640

[E] R. Engelking, General topology, PWN, Warszawa, 1977. MR 58:18316b

[K] C. J. Knight, Box topologies, Quart. J. Math. Oxford 15 (1964), 41-54. MR 28:3398

[L] L. B. Lawrence, Failure of normality in the box product of uncountably many real lines, preprint. CMP 95:04

[vM] J. van Mill, Collected papers of Eric K. van Douwen, preprint.

[R] M. E. Rudin, Lectures on set-theoretic topology, CBMS Regional Conf. Ser. in Math., vol. 23, Amer. Math. Soc., Providence, RI, 1975. MR 51:4128

[W] S. W. Williams, Paracompact sets in box products, preprint.

Department of Mathematics, University of South Carolina, Columbia, South CarOLINA 29208

E-mail address: nyikos@math.sc.edu

Department of Mathematics and Computer Science, Pembroke State University, PemBroke, North Carolina 28372

E-mail address: leszek@nat.pembroke.edu 